\title{
Identification of biomarkers for endometriosis in plasma from patients with endometriosis using a proteomics approach
}

\author{
JIN-HEE HWANG ${ }^{1 *}$, KYU-SUP LEE ${ }^{2 *}$, JONG-KIL JOO ${ }^{2}$, TAO WANG ${ }^{1,3}$, JUNG-BIN SON $^{2}$, \\ JONG-HA PARK ${ }^{2}$, DAE-YOUN HWANG ${ }^{4}$, MAN-HO CHOI $^{5}$ and HONG-GU LEE ${ }^{1}$ \\ ${ }^{1}$ Department of Animal Science and Technology, College of Animal Bioscience and Technology, Konkuk University, \\ Seoul 143-701; ${ }^{2}$ Department of Obstetrics and Gynecology, Pusan National University School of Medicine, Yangsan, \\ Gyeongnam 626-770, South Korea; ${ }^{3}$ College of Animal Science and Technology, Jilin Agricultural University, \\ Changchun, Jilin 130118, P.R. China; ${ }^{4}$ Department of Biomaterial Science, College of Natural Resources and \\ Life Science, Pusan National University, Miryang 627-706; ${ }^{5}$ Future Convergence Research Division, \\ Korea Institute of Science and Technology, Seoul 136-791, South Korea
}

Received August 29, 2013; Accepted April 4, 2014

DOI: $10.3892 / \mathrm{mmr} .2014 .2291$

\begin{abstract}
The present study aimed to examine potential biomarkers for the diagnosis of endometriosis. A plasma-based proteomic approach, including 2-dimentional electrophoresis and mass spectrometry, was used. Samples were obtained from patients with $(n=15)$ and without $(n=15)$ endometriosis, or from mice with surgically induced endometriosis. Seven spots corresponding to six differentially expressed proteins were identified in the human plasma samples. However, only haptoglobin (Hp) was identified to be significantly decreased in the plasma levels of patients with endometriosis $(\mathrm{P}<0.05)$ and in mice with surgically induced endometriosis $(\mathrm{P}<0.05)$. The results demonstrated that $\mathrm{Hp}$ was downregulated in females with endometriosis, and it therefore, may be a useful diagnostic tool as a biomarker of endometriosis.
\end{abstract}

\section{Introduction}

Endometriosis manifests in $\sim 5-20 \%$ of females with pelvic pain, $20-50 \%$ of infertile females and $6-10 \%$ of females of a reproductive age (1). This gynecological disease is defined as the presence of endometrial tissue outside of the uterine cavity, including in the peritoneum, ovaries, fallopian tubes, pleura, lungs or, rarely, the brain (1). The majority of endometriosis cases are diagnosed through direct inspection of

Correspondence to: Dr Hong-Gu Lee, Department of Animal Science and Technology, College of Animal Bioscience and Technology, Konkuk University, 120 Neungdong-ro, Gwangjin-gu, Seoul 143-701, South Korea

E-mail: hglee66@konkuk.ac.kr

${ }^{*}$ Contributed equally

Key words: endometriosis, haptoglobin, plasma, proteomics the abdominal cavity by laparoscopy or laparotomy $(2,3)$. However, these diagnostic approaches are invasive and may not always be feasible. As a result, a number of non-invasive diagnostic tools have emerged, which facilitate the diagnosis of minimal-mild and moderate-severe endometriosis with high sensitivity and clinically accurate specificity. Serum cancer antigen 125 (CA125) has been most widely utilized for the prediction of endometriosis (4); however, a number of reports have suggested that CA125 is not a reliable diagnostic tool for patients $(5,6)$. Aside from CA125, five other plasma biomarkers including interleukin-6 (IL-6), IL-8, tumor necrosis factor- $\alpha$, high-sensitivity C-reactive protein and CA19-9 have also been considered as non-invasive diagnostic biomarkers of endometriosis (7). In addition, a number of other proteins, including anti- $\alpha$-enolase-autoAb, anti-serine/threonine protein kinase-autoAb and anti-syntaxin5-autoAb, have also been detected in the serum of patients with endometriosis (8-10). However, the majority of these proteins lack clinical significance as tools for endometriosis diagnosis. Currently, novel approaches, such as microarrays and proteomics, are emerging as preferred techniques in the study of endometriosis (11-13). A variety of samples, including serum, peritoneal fluid, eutopic and ectopic endometrial tissue, and endometrial fluid have been analyzed using these strategies, which has subsequently facilitated the progression of endometriosis diagnostic research (14-18). In the present study, plasma samples obtained from females with $(n=15)$ and without $(n=15)$ endometriosis were analyzed by proteomic techniques, and then the observed candidate proteins were validated in the plasma of females with endometriosis and mice with surgically induced endometriosis.

\section{Subjects and methods}

Recruited female subjects. This study was approved by the Institutional Review Board at Pusan National University Hospital (PNUH IRB 2010144; Yangsan, Gyeongnam, South Korea) and all experiments involving animals were approved 
by the Animal Care and Use Committee of Pusan National University (PNU-2012-0120). Females with ( $\mathrm{n}=15$; endometriosis) and without ( $\mathrm{n}=15$; control) histologically confirmed endometriosis were enrolled. All the recruited subjects signed an informed consent form prior to their participation in the study. Females in the control group (aged, 25-48) were undergoing laparoscopy or laparotomy for gynecological problems other than endometriosis, i.e., ovarian cysts (mature cystic teratoma, functional cysts and hemorrhagic cysts). The endometriosis group consisted of females (aged, 27-40) who were diagnosed with endometriosis following laparoscopy or laparotomy. These patients complained of abdominal/pelvic pain, dysmenorrhea and/or subfertility. The patients underwent ultrasonography, pelvic CT and/or pelvic MRI in an outpatient clinic. Two patients were classified with minimal to mild disease (stages I and II) and 13 patients with moderate to severe disease (stages III and IV). Blood samples were collected during the proliferative phase from recruited patients. Blood was centrifuged at $400 \mathrm{x}$ g for $15 \mathrm{~min}$ at $4^{\circ} \mathrm{C}$ and then the supernatant was collected and stored at $-80^{\circ} \mathrm{C}$ until assayed.

Murine model of intraperitoneal endometriosis. Seven-week-old female BALB/C mice $(n=16)$ were obtained from Koa Tech, Ltd. (Pyeongtaek, Gyeonggi, South Korea). The mice were housed in cages in environmentally controlled rooms (ambient temperature, $22 \pm 2^{\circ} \mathrm{C}$; relative humidity, $50 \pm 10 \%$ ) under a 12/12 h light-dark cycle (lights on 8:00 am-8:00 pm). Food and water were available ad libitum.

The endometriosis mouse model was established according to the methods previously described (19). Briefly, donor mice were subcutaneously injected with $\beta$-estradiol in corn oil (100 mg/kg; Sigma-Aldrich, St. Louis, MO, USA). One week later, donor mice were anesthetized by intraperitoneal injection of Zoletil (weight, $15 \mathrm{mg} / \mathrm{kg}$; Virbac, Carros, France) and 2\% xylazine (Rompun; 15 mg/kg body weight; Bayer, Leverkusen, Germany). The entire uterus was then removed and transferred to a petri dish containing saline. Isolated uterine horns were reduced to small fragments with scissors, which were then resuspended in saline. Half of the preparation was injected into the pertitoneum of two anesthetized recipient mice with a syringe (day 0). Then, the incision was closed with running 4-0 prolene muscle and skin sutures. Following surgery, recipient mice ( $n=8$; endometriosis) were injected with $\beta$-estradiol in corn oil ( $5 \mathrm{mg} / \mathrm{kg}$; Sigma-Aldrich) every week, whereas control mice $(n=4)$ were treated with corn oil only.

Two weeks later, all mice were sacrificed by $\mathrm{CO}_{2}$ asphyxiation. Blood samples were collected into tubes coated with heparin and the plasma was obtained following centrifugation at $400 \mathrm{x} \mathrm{g}$ for $15 \mathrm{~min}$. Aliquots of plasma samples were frozen $\left(-80^{\circ} \mathrm{C}\right)$ until analyzed. In addition, the uterine horns and endometriotic lesions were collected and fixed in formalin for three days for the following histological evaluation. Subsequent to fixation, mice endometrial lesions and uterine horns were imbedded in paraffin wax and sectioned into $5-\mu \mathrm{m}$ thick slices. The sections were stained with hematoxylin and eosin (Sigma-Aldrich). Stained sections were observed by light microscopy (CX31, Olympus, Tokyo, Japan).

Protein identification and 2-dimentional electrophoresis $(2-D E)$. Albumin and IgGs were removed from the human plasma using a Multiple Affinity Removal System Spin Cartridge HAS/IgG (Agilent Technologies, Wilmington, DE, USA). Following this, the samples were concentrated using a Spin Concentrator (Agilent Technologies) according to the manufacturer's instructions. The total protein concentrations of the samples were determined using a bicinchoninic acid assay kit (Pierce Biotechnology, Inc., Rockford, IL, USA). Electrophoretic separation of the proteins was performed as previously described (20). Briefly, $100 \mu \mathrm{g}$ proteins diluted in isoelectric focusing buffer was loaded onto $\mathrm{pH}$ 3-10 NL Immobiline DryStrip gels (18 cm; GE Healthcare, Piscataway, NJ, USA). The strips were then equilibrated for $15 \mathrm{~min}$ in an equilibration buffer [50 mM Tris- $\mathrm{HCl}(\mathrm{pH} 8.8), 6 \mathrm{M}$ urea, $2 \%$ sodium dodecyl sulfate (SDS), $30 \%$ glycerol and $0.002 \%$ (w/v) bromophenol blue] containing 1\% dithiothreitol or $135 \mathrm{mM}$ iodoacetamide, separately. The equilibrated strips were inserted into $12 \%$ SDS-polyacrylamide gel electrophoresis (PAGE) gels $(18 \mathrm{~cm})$ in an Ettan DALT 2-D gel system (GE Healthcare) and the gels were stained using a PlusOne Silver Staining kit (GE Healthcare). The spots were analyzed by ProteomWeaver software 2.2 (Definiens AG, Munich, Germany). Spots exhibiting differences in staining intensity of at least 2-fold were selected for electrospray ionization-quadrupole-time of flight/mass spectrometer (ESI-Q-TOF/MS) analysis. The details of ESI-Q-TOF/MS analysis have been described in a previous study (21).

Western blotting. Each human or mouse (10 or $80 \mu \mathrm{g})$ sample was separated by 8 or $10 \%$ SDS-PAGE and then transferred onto nitrocellulose membranes using a Trans-blot ${ }^{\circledR}$ SD Semi-dry Transfer Cell (Bio-Rad, Hercules, CA, USA) for $30 \mathrm{~min}$. The nitrocellulose membrane (Whatman, Dassel, Germany) was immediately blocked with a 5\% non-fat milk solution at room temperature for $1 \mathrm{~h}$. The membrane was then incubated overnight at $4^{\circ} \mathrm{C}$ with the appropriate primary antibodies: Anti- $\alpha-2$ macroglobin (1:1000; Abcam, Cambridge, MA, USA), anti-apolipoprotein E (1:1000; Abcam), anti-apolipoprotein L1 (1:1000; Abcam), anti-C4A (1:5000; Santa Cruz Biotechnology, Inc., Santa Cruz, CA, USA), anti-haptoglobin (HP) protein (1:250000; Abcam) and anti-leucine-rich $\alpha$-2-glycoprotein precursor (LRG; 1:1000; Abcam). Following this, membranes were washed three times with $1 \mathrm{X}$ Tris-buffered saline with Tween-20 and incubated (for $20 \mathrm{~min}$ at room temperature) with the following secondary antibodies: Anti-rabbit IgG-horseradish peroxidase (HRP) (1:5000 or 1:1000, Thermo Fisher Scientific, Inc., Rockford, USA) and anti-mouse IgG-HRP (1:5000; Abcam). The proteins on the membrane were visualized using an enhanced chemiluminescence detection kit (Surmodics, Eden Prairie, MN, USA). Bands were quantified using Image J 1.43 software (http://rsb.info.nih.gov/ij/download.html) and protein levels were normalized to those of $\beta$-actin on the same membrane.

Statistical analysis. All results were expressed as the mean \pm standard error of the mean. A comparison between the two groups was performed using Student's t-test and Tukey's HSD Kramer comparison test. $\mathrm{P}<0.05$ was considered to indicate a statistically significant difference. All statistical analyses were performed using JMP 7.0.1 (SAS Institute Inc., Cary, NC, USA). 
Table I. List of plasma protein spots identified by ESI-Q-TOF/MS.

\begin{tabular}{lllllll}
\hline Spot no. & Accession no. & Protein name & Score & MW $(\mathrm{kDa} / \mathrm{p} I)$ & Expression $^{\mathrm{a}}$ & Function \\
\hline 1 & gil114039 & APOE & 201 & $36.2 / 5.65$ & Down & Lipoprotein particle mediator \\
2 & gil34782950 & C4A protein & 115 & $32.1 / 8.52$ & Down & Innate immune response \\
3 & gil16418467 & LRG & 236 & $38.2 / 6.45$ & Down & Signal transduction \\
4 & gil3337390 & Hp & 673 & $38.7 / 6.14$ & Down & Cellular homeostasis \\
5 & gil177870 & $\alpha-2 \mathrm{M}$ & 110 & $32.1 / 8.52$ & Down & Protein inhibitor \\
6 & gil16418467 & LRG & 139 & $38.2 / 6.45$ & Down & Signal transduction \\
7 & gil12232634 & APOL1 & 41 & $42.4 / 6.37$ & Down & Innate immune response
\end{tabular}

${ }^{\mathrm{a}}$ Vs. control. Apo-E, apolipoprotein-E precursor; LRG, leucine-rich alpha 2-glycoprotein precursor; Hp, haptoglobin; $\alpha$-2M, $\alpha$-2-macroglobulin precursor; LRG, leucine-rich alpha 2-glycoprotein precursor; Apo-L1, apolipoprotein-L1 precursor; MW, molecular weight; ESI-Q-TOF/MS, electrospray ionization-quadrupole-time of flight/mass spectrometer.
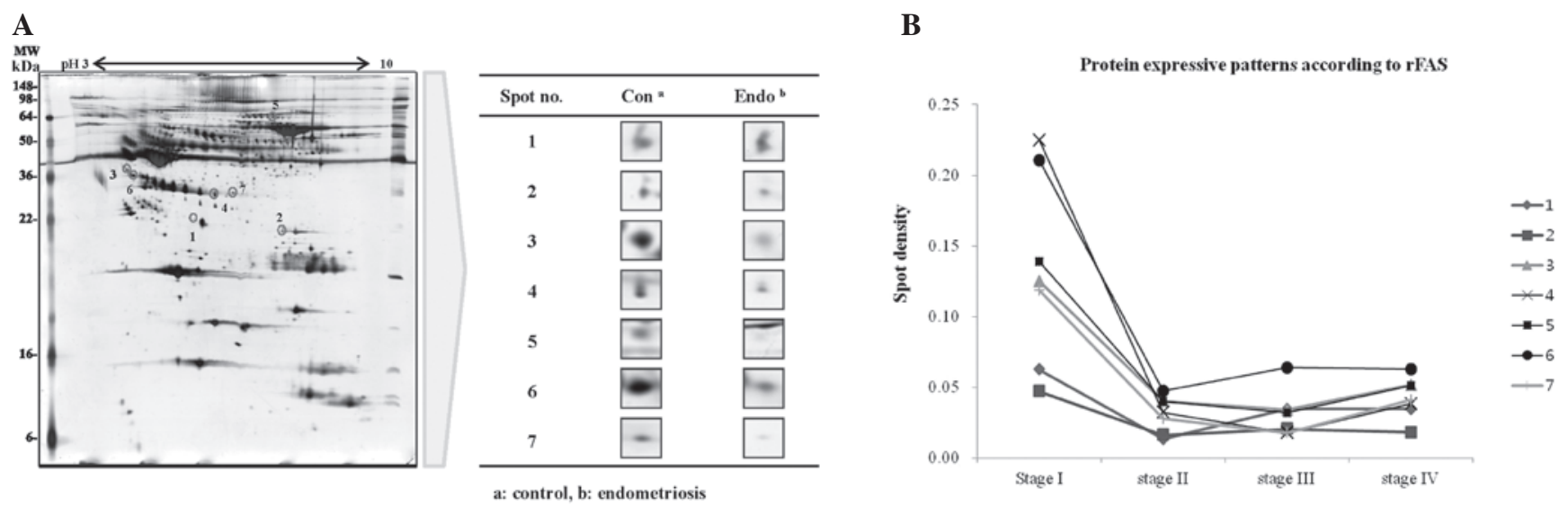

Figure 1. Proteomic analysis of highly expressed proteins in the plasma of females with endometriosis. (A) Representative gel based on proteins pooled from females with or without endometriosis $(n=30)$. The table indicates protein spots revealing differences in the expression of at least 2 -fold in the control compared with endometriosis patients. (B) Seven spots expressed downregulation from stages I to IV.

\section{Results}

Proteomic analysis of plasma proteins in females with endometriosis. Plasma samples from endometriosis and control patients were pooled into two groups and the differences in spot density within 2-DE maps were determined using ProtemoWeaver software (Definiens AG). A total of 220 protein spots in the endometriosis group $(n=15)$ and 203 spots in the control group $(n=15)$ were observed, of which 25 of these spots were identified to have differences in spot density of at least 2-fold. Twenty spots in the endometriosis samples had lower densities, whereas five spots were detected only in the control. Seven spots were then selected after the densities comparison followed the clinical stage (stage $\mathrm{I}=1$, stage $\mathrm{II}=1$, stage III=3, stage IV=10) of endometriosis. (Fig. 1). Finally, they were identified as Hp, LRG, C4A protein, apolipoprotein E precursor (Apo-E), apolipoprotein L1 precursor (ApoL-1) and alpha-2-macroglobulin precursor $(\alpha-2 \mathrm{M})$ by ESI-Q-TOF/MS (Table I).

Validation of biomarkers by western blotting. Western blotting was performed on a total of 13 samples including from females with endometriosis (early stage $=2$, advanced stage $=7$ ) and the control group $(n=4)$. Quantification of protein densities was conducted by Image $\mathrm{J}$ software (Fig. 2).

As demonstrated in Fig. 2B, the relative densities of $\mathrm{Hp}$, ApoL-1 and LRG in the endometriosis group were decreased compared with those in the the control group. In particular, quantitation of the $\mathrm{Hp}$ expression was reduced 3.05-fold compared with that of the control $(\mathrm{P}<0.05)$. By contrast, there was no change in Apo-E expression in females with endometriosis compared with those without. $\mathrm{C} 4 \mathrm{~A}$ and $\alpha-2 \mathrm{M}$ protein expression in females with endometriosis generally increased compared with that in the control group. LRG expression gradually decreased from the early to advanced stages.

Fig. 2C demonstrates the results of early (stages I and II) and advanced (stages III and IV) stage groups when compared with the control, in patients with and without endometriosis. The relative densities of $\mathrm{Hp}$ were reduced in early and advanced stages compared with the control $(\mathrm{P}<0.05)$. The expression of ApoL-1 generally decreased in the early and advanced stage groups compared with the control group. However, the expression of ApoL-1 in the advanced stage group increased compared with the early stage. The relative density of Apo-E was not observed to be altered in each 
A

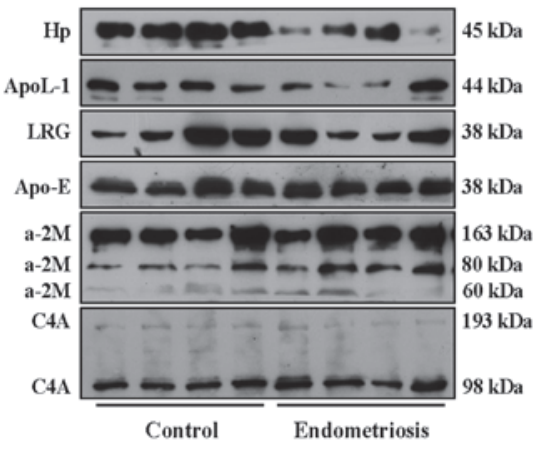

B

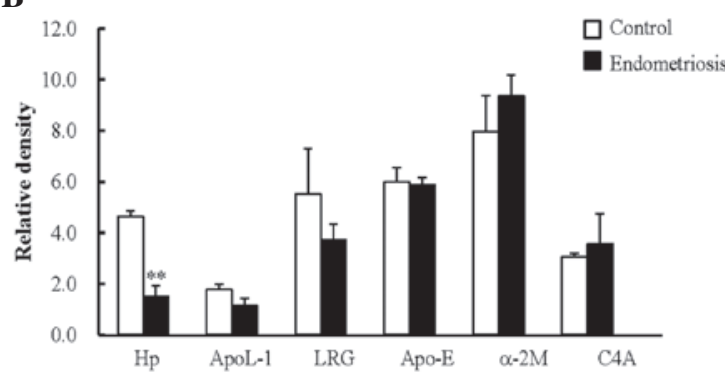

C

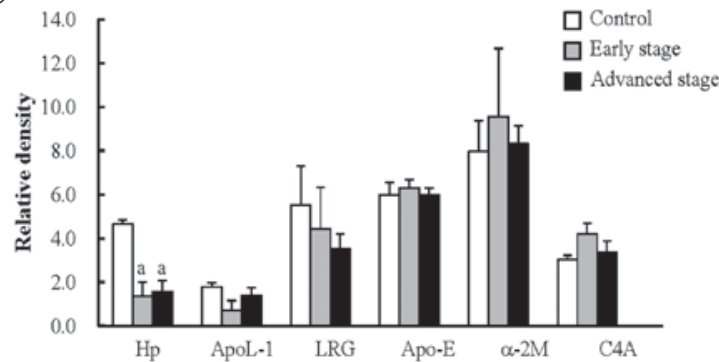

Figure 2. Validation of plasma proteins by western blotting. (A) Western blot analysis of 13 plasma samples probed with anti-Hp, anti-ApoL-1, anti-LRG, anti-Apo-E, anti- $\alpha-2 \mathrm{M}$ and anti-C4A antibodies. (B) The relative densities of proteins in females with endometriosis compared with those without endometriosis. (C) Early stage indicates stages I and II and advanced stage indicates stages III and IV. Values are expressed as the mean \pm standard error of the mean. ${ }^{* *} \mathrm{P}<0.01$ vs. control (Student's t-test) and ${ }^{a} \mathrm{P}<0.05$ vs. control (Tukey's HSD Kramer test). Apo-E, apolipoprotein-E precursor; LRG, leucine-rich alpha 2-glycoprotein precursor; Hp, haptoglobin; $\alpha$-2M, alpha-2-macroglobulin precursor; Apo-L1, apolipoprotein-L1 precursor.
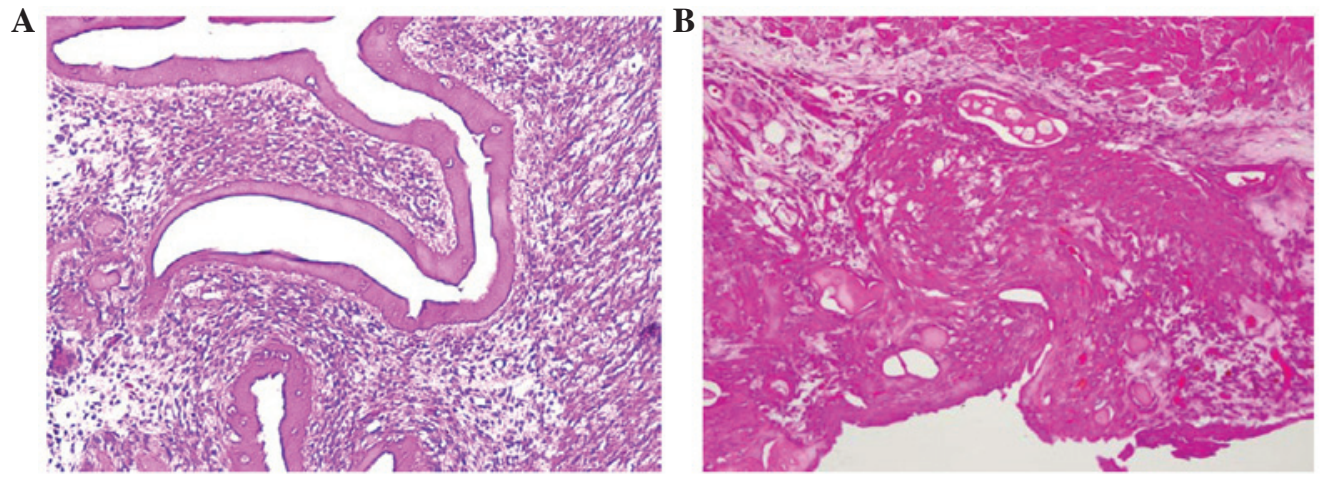

Figure 3. Histology of mouse endometriotic lesion and eutopic uterus. The mice underwent surgical induction of endometriosis. (A) Eutopic uteri and (B) endometriotic lesions were extracted 14 days later. Representative hemotoxylin and eosin-stained sections demonstrate typical histological endometriotic lesion (original magnification, $\mathrm{x} 200$ ).
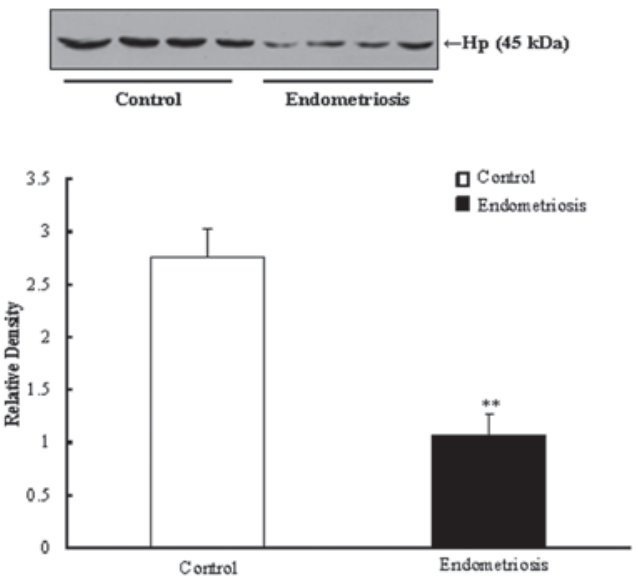

Figure 4. Confirmation of a potential marker in mouse plasma using western blot analysis. Values are expressed as the mean \pm standard error of the mean. ${ }^{* *} \mathrm{P}<0.01$ vs. control (Student's t-test). group. The levels of $\alpha-2 \mathrm{M}$ and $\mathrm{C} 4 \mathrm{~A}$ increased in the early and advanced stages compared with the controls.

Verification of biomarkers of endometriosis in mouse plasma. Histological examination revealed that uterine tissue samples at 14 days following transplantation into the peritoneal cavity had developed into endometriotic lesions with a typical histological appearance (Fig. 3). As illustrated in Fig. 4, the plasma Hp level was significantly downregulated in the surgically induced mouse model, as compared with the control mice $(\mathrm{P}<0.01)$.

\section{Discussion}

Endometriosis is defined as the presence of proliferating, functional, endometriotic-like tissue outside of the uterine cavity (22). A definite diagnosis of the disease relies on inspection of endometriotic lesions with histological confirmation 
by laparoscopy or laparotomy. Additionally, transvaginal ultrasound or specific biomarkers, such as CA-125, have been utilized as diagnostic strategies. However, these methods are not diagnostically useful, due to their relatively low sensitivity and specificity in the early stages of the disease $(23,24)$. Therefore, in the present study, the disease was investigated on a molecular level by analyzing the plasma of females with and without endometriosis. The six identified proteins included $\alpha-2 \mathrm{M}, \mathrm{Hp}$, ApoL-1, LRG precursor, Apo-E precursor and C4A protein. These proteins have a variety of functions, involving cellular homeostasis, immune response, apoptosis regulation and signal transduction. In the present study, only $\mathrm{Hp}$ was identified by western blotting analysis. The expression of this protein was significantly lower level in plasma samples from patients with endometriosis compared with controls. Downregulation of $\mathrm{Hp}$ in plasma is disease-specific and since $\mathrm{Hp}$ is an acute-phase protein and is usually induced in the periphery as a marker of inflammation, it may be a distinguishing factor of inflammatory disease (25). Cocciolo et al (26) demonstrated that the Hp $\beta$-chain is significantly downregulated and oxidatively modified in Alzheimer's disease. Oxidative post-synthetic modifications lead to Hp $\beta$-chain dysfunction and may be correlated with disease pathology. Reactive oxygen species (ROS)-induced protein modifications consequently alter protein function and antigenecity and are therefore implicated in immunological deleterious reactions associated with inflammatory and/or autoimmune injury $(27,28)$. This oxidative stress mechanism may have an important role in the development and progression of endometriosis (29). However, the correlation between decreased expression and oxidation of the Hp protein, and oxidative stress in endometriosis has not been reported, therefore, further studies are required to further elucidate this.

In the present study a surgically-induced endometriosis mouse model was successfully established. The induction of endometriosis-like lesions in mice was performed by sygeneic and autologous transplantation of uterine tissue into the peritoneal cavity, as spontaneous development of endometriosis is dependent on menstruation. In this model, endometriosis-like lesions demonstrated a histopathology similar to that observed in human endometriotic lesions. Therefore, our mouse model reliably represented the histomorphology of human endometriosis. Hp identified in human plasma was also downregulated in this surgically-induced endometriosis murine model. These data imply that Hp may be a disease-specific protein in endometriosis.

In conclusion, six differentially expressed proteins were identified in the plasma of females with endometriosis. However, only Hp was identified to be significantly decreased in the plasma of endometriosis patients and in the surgically-induced murine model. Therefore, Hp may be used as a potential biomarker for endometriosis diagnostic strategies for the future. However, further studies are required to evaluate its clinical utility.

\section{Acknowledgements}

This study was supported by a grant of the Korea Health Technology R\&D Project, Ministry of Health \& Welfare, South Korea (no. A 101367) and the Next-Generation BioGreen 21 Program (no. PJ00819105), Rural Development Administration, South of Korea.

\section{References}

1. Sasson IE and Taylor HS: Stem cells and the pathogenesis of endometriosis. Ann NY Acad Sci 1127: 106-115, 2008.

2. Ozkan S, Murk W and Arici A: Endometriosis and infertility: epidemiology and evidence-based treatments. Ann NY Acad Sci 1127: 92-100, 2008.

3. Bulun SE: Endometriosis. N Engl J Med 360: 268-279, 2009.

4. Audebert A: Endometriosis coaching. Gynecol Obstet Fertil 34: 329-336, 2006 (In French).

5. Bast RC Jr, Xu FJ, Yu YH, Barnhill S, Zhang Z and Mills GB: CA 125: the past and the future. Int J Biol Marker 13: 179-187, 1998.

6. Chen FP, Soong YK, Lee N and Lo SK: The use of serum CA-125 as a marker for endometriosis in patients with dysmenorrhea for monitoring therapy and for recurrence of endometriosis. Acta Obstet Gynecol Scand 77: 665-670, 1998.

7. Mihalyi A, Gevaert O, Kyama CM Simsa P, Pochet N, De Smet F, De Moor B, Meuleman C, Billen J, Blanckaert N, Vodolazkaia A, Fulop V and D'Hooghe TM: Non-invasive diagnosis of endometriosis based on a combined analysis of six plasma biomarkers. Hum Reprod 25: 654-664, 2010.

8. Nabeta M, Abe Y, Kagawa L Haraguchi R, Kito K, Ueda N, Sugita A, Yokoyama M, Kusanagi Y and Ito M: Identification of anti- $\alpha$-enolase autoantibody as a novel serum marker for endometriosis. Proteomics Clin Appl 3: 1201-1210, 2009.

9. Nabeta M, Abe Y, Haraguchi R, Kito K, Kusanagi Y and Ito M: Serum anti-PDIK1L autoantibody as a novel marker for endometriosis. Fertil Steril 94: 2552-2557, 2010.

10. Nabeta M, Abe Y, Takaoka Y, Kusanagi Y and Ito M: Identification of anti-syntaxin 5 autoantibody as a novel serum marker of endometriosis. J Reprod Immunol 91: 48-55, 2011.

11. Eyster KM, Boles AL, Brannian JD and Hansen KA: DNA microarray analysis of gene expression markers of endometriosis. Fertil Steril 77: 38-42, 2002.

12. Arimoto T, Katagiri T, Oda K Tsunoda T, Yasugi T, Osuga Y, Yoshikawa H, Nishii O, Yano T, Taketani Y and Nakamura Y: Genome-wide cDNA microarray analysis of gene-expression profiles involved in ovarian endometriosis. Int $\mathrm{J}$ Oncol 22: 551-560, 2003.

13. Kao LC, Germeyer A, Tulac S Lobo S, Yang JP, Taylor RN, Osteen K, Lessey BA and Giudice LC: Expression profiling of endometrium from women with endometriosis reveals candidate genes for disease-based implantation failure and infertility. Endocrinology 144: 2870-2881, 2003.

14. Gupta S, Agarwal A, Sekhon L, Krajcir N, Cocuzza M and Falcone T: Serum and peritoneal abnormalities in endometriosis: potential use as diagnostic markers. Minerva Ginecol 58: 527-551, 2006.

15. Zhang H, Niu Y, Feng J, Guo H, Ye X and Cui H: Use of proteomic analysis of endometriosis to identify different protein expression in patients with endometriosis versus normal controls. Fertil Steril 86: 274-282, 2006.

16. Ferrero S, Gillott DJ, Remorgida V, Anserini P, Leung KY, Ragni N and Grudzinskas JG: Proteomic analysis of peritoneal fluid in women with endometriosis. J Proteome Res 6: 3402-3411, 2007.

17. Fowler PA, Tattum J, Bhattacharya S, Klonisch $\mathrm{T}$, Hombach-Klonisch S, Gazvani R, Lea RG, Miller I, Simpson WG and Cash P: An investigation of the effects of endometriosis on the proteome of human eutopic endometrium: a heterogeneous tissue with a complex disease. Proteomics 7: 130-142, 2007.

18. Ametzazurra A, Matorras R, García-Velasco JA Prieto B, Simón L, Martínez A and Nagore D: Endometrial fluid is a specific and non-invasive biological sample for protein biomarker identification in endometriosis. Hum Reprod 24: 954-965, 2009.

19. Somigliana E, Viganò P, Rossi G, Carinelli S, Vignali M and Panina-Bordignon P: Endometrial ability to implant in ectopic sites can be prevented by interleukin-12 in a murine model of endometriosis. Hum Reprod 14: 2944-2950, 1999.

20. Wang T, Lee HG, Hwang JH, Oh JJ, Lim JN, Kang HS, Joo JK and Lee KS: Myoglobin: a promising exogenous reference marker using in proteomics analysis. Food Sci Biotechnol 22: 393-398, 2013.

21. Hwang JH, Oh JJ, Wang T, Jin YC, Lee JS, Choi JR, Lee KS, Joo JK and Lee HG: Identification of biomarkers for endometriosis in eutopic endometrial cells from patients with endometriosis using a proteomics approach. Mol Med Rep 8: 183-188, 2013. 
22. Galle PC: Clinical presentation and diagnosis of endometriosis. Obstet Gynecol Clin North Am 16: 29-42, 1989.

23. Mol BW, Bayram N, Lijmer JG Wiegerinck MA, Bongers MY, van der Veen F and Bossuyt PM: The performance of CA-125 measurement in the detection of endometriosis: a meta-analysis. Fertil Steril 70: 1101-1108, 1998.

24. Moore J, Copley S, Morris J, Lindsell D, Golding S and Kennedy S: A systematic review of the accuracy of ultrasound in the diagnosis of endometriosis. Ultrasound Obstet Gynecol 20: 630-634, 2002

25. Slobodianik NH, Feliu MS, Perris P Barbeito S, Strasnoy I, Franchello A and Ferraro M: Inflammatory biomarker profile in children with cystic fibrosis: preliminary study. Proc Nutr Soc 69: 354-356, 2000.
26. Cocciolo A, Di Domenico F, Coccia R Fiorini A, Cai J, Pierce WM, Mecocci P, Butterfield DA and Perluigi M: Decreased expression and increased oxidation of plasma haptoglobin in Alzheimer disease: Insights from redox proteomics. Free Radic Biol Med 53: 1868-1876, 2012.

27. Iborra A, Palacio JR and Martinez P: Oxidative stress and autoimmune response in the infertile woman. Chem Immunol Allergy 88: 150-162, 2005.

28. Sohal RS: Role of oxidative stress and protein oxidation in the aging process. Free Radic Biol Med 33: 37-44, 2002.

29. Carvalho LF, Samadder AN, Agarwal A, Fernandes LF and Abrão MS: Oxidative stress biomarkers in patients with endometriosis: systematic review. Arch Gynecol Obstet 286: 1033-1040, 2012. 